\title{
Long-term Survival in Patients Treated with a Robotic Radiosurgical Device for Liver Metastases
}

\author{
Sebastian Stintzing, MD \\ Jobst von Einem, MD' \\ Christoph Fueweger, $\mathrm{PhD}^{2}$ \\ Alfred Haidenberger, MD2 \\ Michael Fedorov, MD2 \\ Alexander Muavcevic, MD2
}

${ }^{1}$ Department of Medicine III, University

Hospital, LMU Munich, Munich,

${ }^{2}$ European Cyberknife Center Munich,

Munich, Germany

Correspondence: Sebastian Stintzing, MD

Department of Medicine III, University

Hospital, LMU Munich, Marchioninistr. 15,

81377 Munich, Germany

Tel: $49-89-4400-75126$

Fax: 49-89-4400-75124

E-mail: sebastian.stintzing@med.uni-muenchen.de

Received December 15, 2017

Accepted April 13, 2018

Published Online April 16, 2018

\begin{abstract}
Purpose
The treatment of liver metastases with local procedures is a fast progressing field. For the most, long-term survival data is missing raising questions with regard to the efficacy of such modalities when compared to surgical resection. Radiosurgery using the Cyberknife device enables the treatment of liver lesions with a single-session approach. Here we present longterm survival data to explore the curative potential of this strategy.
\end{abstract}

\section{Materials and Methods}

Patients with oligo-metastatic disease limited to the liver have been treated with single-session or hypo-fractioned radiosurgery in curative intent and prospectively followed until death. Follow-up (FU) was performed using magnetic resonance imaging (MRI) 2 months after radiation and at 3-month intervals for the first 2 years. After that annual computed tomography or MRI scans were performed until 5 years post-treatment. Local recurrence in the radiated volume and recurrence outside the treated volume were used to define local and distant progression. Survival times were censored at the time of the last FU.

\section{Results}

One hundred twenty-six patients treated between 2005 and 2015 with 194 lesions were included into this study. Median FU was 30.0 months. According to Response Evaluation Criteria in Solid Tumors, $55.2 \%$ had a complete remission and $11.3 \%$ a partial remission. Seventy-two point two percent recurred outside the radiated lesion and median overall survival was 35.2 months with a 3 -year survival rate of $47.7 \%$.

\section{Conclusion}

This is currently the largest cohort of stereotactic body radiation therapy treated liver lesions with a median long-term follow of 30 months. Robotic radiosurgery using a single session approach has a high efficacy to control the radiated lesion with the potential to cure patients.

\section{Introduction}

Patients with metastatic disease dominant or limited to the liver benefit from aggressive local treatment in terms of overall survival (OS) times. This has been demonstrated particularly for colorectal liver metastases (CRLM) where the secondary resection of liver metastases has shown to prolong survival significantly [1]. The actual European Society of Med-
Key words

Radiosurgery, Radiation, Liver metastases, Radiosurgery,

Colonic neoplasms, Liver 
(microwave [MW] ablation, radio-frequency ablation [RFA], or laser-induced thermotherapy), stereotactic body radiation (SBRT), or selective internal radiation as the "toolbox" that should be utilized according to the experience of the respective treatment center [2]. The efficacy of those local treatment modalities is seen as comparable to each other even so no formal comparisons in clinical trials have been carried out so far. As recurrence rates after surgery of liver metastases are between $50 \%$ and $75 \%$ within the first 2 years after surgery [3-5], surgery in curative intent is not perfect when it comes to disease control and is biased by patient selection. Retrospective analyses on RFA versus surgery suggest a superior outcome of surgery [3] in CRLM [6]. For hepatocellular carcinomas retrospective analyses showed a better outcome with respect to disease-free survival but comparable results for OS when RFA was compared to surgery [7]. For thermal ablation modalities the size of the treated lesion has been shown to be of critical value with respect to survival and recurrence [8] favoring tumors with a diameter of less than $3 \mathrm{~cm}$. The advantage of external body radiation is mainly seen in the absence of an invasive procedure promising less morbidity with comparable efficacy to other local treatments. Moreover, systemic treatment may not be interrupted during SBRT. The feasibility of SBRT has been shown by the landmark paper of Rusthoven et al. [9] where 63 lesions have been treated with SBRT with a 2-year local control rate of $100 \%$ and a median OS of 20.5 months. Technical difficulties that have to be overcome prior to radiate a moving organ such as the liver are to track the lesion during radiation and to re-focus the radiation beam to the target lesion. This is pivotal to maximize efficacy and to minimize adverse events. There are different techniques to ensure those requirements such as magnetic resonance imaging (MRI) and computed tomography $(\mathrm{CT})$ scan based planning procedures in combination of immobilization devices $[10,11]$. Next to SBRT the development of intensity modulated radiotherapy (IMRT) enabled the radiation oncologist to modulate the intensity of the radiation beam to spare healthy tissue. The CyberKnife technique consists of a linear accelerator (LINAC) mounted on a six-axis robotic manipulator that allows radiation of the target lesion from multiple angles, track the lesion in real time and adjust the beam accordingly. This enables single session robotic real time tracking without the need of an immobilization device [12].

Here, we present the long-term outcome of patients treated in curative intent with radiation of liver lesions using the CyberKnife system.

\section{Materials and Methods}

Patients treated at the European Cyberknife Center Munich between 2005 and 2015 in curative intent for liver lesions were prospectively followed until death. All patients were evaluated prior to radiation by a multidisciplinary tumor board consisting of medical oncologists, hepato-biliary surgeons, interventional radiologists and radiation oncologists. All patients gave informed consent prior treatment for follow-up (FU) procedure and telephone contacts. FU was performed using MRI scanning 2 months after radiation and at 3-month intervals for the first 2 years or at the recommendation of the medical oncologist.

\section{Treatment}

CyberKnife treatment was performed as described before [12]. In short, a radiosurgical device (Accuray, Sunnyvale, CA) with real time tumor tracking was used. Prior to radiation, one or two gold fiducials (CP Medical Inc., Portland, OR) were placed inside or next to the metastases via an 18G needle under local anesthesia and CT fluoroscopy guidance in the interventional radiology unit. Contrast enhanced dynamic CT (arterial and portalvenous phase) and MRI (using liver-specific contrast agents) were used to identify 3D target volume and to exclude additional intrahepatic tumors. Additionally, a safety margin of $6 \mathrm{~mm}$ was added to the tumor diameter in all three dimensions. In a single session treatment, a total of 24-26 Gy to the 70\% isodose (dose maximum 34-37 Gy) were administered to the target volume, using a 6MV compact LINAC mounted on a six-axis robotic manipulator. In treatment planning, normal tissue constraints suggested by AAPM Task Group 101 [13] for singleand three-fraction SBRT were observed. During treatment, respiratory tumor motion was actively compensated by dynamic Synchrony tracking [14]: a correlation model between a continuous breathing signal from optical markers (attached to the chest) and internal fiducial positions (derived from periodic stereoscopic X-ray images) was established. This information was used by the robotic arm to actively guide all beams to the current location of the lesion. All patients were pretreated with ondansetrone on the day of treatment to prevent nausea due to radiation.

\section{Statistics}

Statistical analysis was performed using IBM SPSS statistics software (SPSS ver. 23, IBM Corp., Armonk, NY). Duration of local control, progression-free survival (PFS) and OS were analyzed using Kaplan-Meier estimation and medians were compared using log-rank test. Time intervals were 
Table 1. Patient characteristics

\begin{tabular}{|c|c|c|c|}
\hline Characteristic & $\begin{array}{c}\text { All } \\
(n=126)\end{array}$ & $\begin{array}{l}\text { Colorectal } \\
\quad(n=71)\end{array}$ & $\begin{array}{l}\text { Non-colorectal } \\
\quad(n=55)\end{array}$ \\
\hline Age, median (range, yr) & $65.4(33-87)$ & $67.1(33-84)$ & $63.4(44-87)$ \\
\hline Female sex & $54(42.9)$ & $16(22.5)$ & $38(69.1)$ \\
\hline \multicolumn{4}{|l|}{ Tumor entities } \\
\hline Colorectal cancer & $71(56.3)$ & $71(100)$ & - \\
\hline Gastrointestinal, non-CRC & $13(10.3)$ & - & $13(23.6)$ \\
\hline Breast & $14(11.1)$ & - & $14(25.5)$ \\
\hline Uro-genital & $15(11.9)$ & - & $15(27.3)$ \\
\hline Bronchial & $5(4.0)$ & - & $5(9.1)$ \\
\hline Liver & $1(0.8)$ & - & $1(1.8)$ \\
\hline Others & $8(6.3)$ & - & $8(14.5)$ \\
\hline Systemic pretreatment & $103(81.7)$ & $62(87.3)$ & $41(74.5)$ \\
\hline Local pretreatment & $95(75.4)$ & $53(74.6)$ & $42(76.4)$ \\
\hline Toolbox & $33(26.2)$ & $18(25.4)$ & $15(27.3)$ \\
\hline Thermal ablation (RFA, MW) & $13(10.3)$ & $10(14.1)$ & $3(5.5)$ \\
\hline SBRT & $13(10.3)$ & $6(8.5)$ & $7(12.7)$ \\
\hline SIRT & $7(5.6)$ & $2(2.8)$ & $5(9.1)$ \\
\hline Liver surgery & $85(67.5)$ & $46(64.8)$ & $39(70.9)$ \\
\hline Follow-up (95\% CI, mo) & $30(19.3-40.8)$ & $34.7(22.3-47.2)$ & $28(4.9-51.1)$ \\
\hline
\end{tabular}

Values are presented as number $(\%)$ unless otherwise indicated. CRC, colorectal cancer; RFA, radiofrequency ablation; MW, microwave ablation; SBRT, stereotactic body radiation treatment; SIRT, selective internal radiation therapy; CI, confidence interval.

measured from the day of radiation until date of progression or death. FU period was calculated using inverse OS method. Local progression was defined as any progression within the treated liver volume whereas overall progression has been defined as any progression at any site. Response as best-overall-response was measured for the treated lesions according to Response Evaluation Criteria in Solid Tumors (RECIST) ver. 1.0 criteria.

\section{Ethical statement}

This study was approved by the Institutional Review Board of the Ethics Committee of the Ludwig-MaximiliansUniversity (LMU) Munich, Germany with a waiver of informed consent (IRB No. 383-08) and performed in accordance with the principles of the Declaration of Helsinki.

\section{Results}

A total of 134 patients with 203 liver lesions were treated between 2005 and 2015 at the European Cyberknife Center
Munich. Of those eight patients were lost to FU. The total number of patients analyzed in this study was 126 with a total of 194 liver lesions that were treated in 145 treatment sessions. Side effects were minor with two Common Terminology Criteria for Adverse Events (CTCAE)'s grade 3 (gastric ulcers) and two CTCAE's grade 2 (nausea); one patient developed a non-symptomatic icterus. The patients with grade 3 gastric ulcer related to cyberknife treatment were treated among the first patients. In detail they were as follows. (1) A 72-year-old woman with sarcoma: total dose to the $70 \%$ isodose, $24 \mathrm{~Gy}$; volume of the lesion, $22.3 \mathrm{cc}$ and Dmax stomach, 22.5 Gy; D 1cc, 18.5Gy. The liver lesion was located in direct proximity to the small curvature of the stomach. A radiation of parts of the stomach was not avoidable and (2) a 67-year-old man with colorectal liver metastasis: total dose to the $70 \%$ isodose, $24 \mathrm{~Gy}$; volume of the lesion $14.3 \mathrm{cc}$ and Dmax stomach, $17 \mathrm{~Gy}$ in close temporal relation to 5-FU and oxaliplatin based chemotherapy. Baseline characteristics are given in Table 1 . In short, $81.7 \%$ of the patients had any kind of systemic chemotherapy prior to the radiation and $75.4 \%$ had any kind of local treatment to the liver, including liver surgery prior to radiation treatment. Most of the lesions were CRLM (56.3\%) with other gastrointestinal tumors, breast and urogenital being the second most fre- 
Table 2. Liver lesions ( $\mathrm{n}=194)$

\begin{tabular}{|c|c|c|c|}
\hline & $\begin{array}{c}\text { Total } \\
(n=194)\end{array}$ & $\begin{array}{c}\text { Colorectal } \\
\text { lesions }(n=113)\end{array}$ & $\begin{array}{l}\text { Non-colorectal } \\
\text { lesions }(\mathrm{n}=\mathbf{8 1})\end{array}$ \\
\hline \multicolumn{4}{|l|}{ Size by diameter $(\mathrm{cm})$} \\
\hline$<3$ & $56(28.9)$ & $29(25.7)$ & $27(33.3)$ \\
\hline $3-5$ & $115(59.3)$ & $66(58.4)$ & $49(60.5)$ \\
\hline$>5$ & $23(11.9)$ & $18(15.9)$ & $5(6.2)$ \\
\hline \multicolumn{4}{|l|}{ Fractions } \\
\hline Single session & $185(95.4)$ & $105(92.9)$ & $80(98.8)$ \\
\hline Hypofractioned & $9(4.6)$ & $8(7.1)$ & $1(1.2)$ \\
\hline No. of treatments & 145 & 80 & 65 \\
\hline \multicolumn{4}{|l|}{ Targets per treatment } \\
\hline 1 & $110(75.9)$ & $59(73.8)$ & $51(78.5)$ \\
\hline 2 & $31(21.4)$ & $18(22.5)$ & $13(20.0)$ \\
\hline 3 & $3(2.1)$ & $3(3.8)$ & - \\
\hline 4 & $1(0.7)$ & - & $1(1.5)$ \\
\hline Volume, median (range, $\mathrm{cm}^{3}$ ) & $21.5(0.6-166)$ & $25.3(0.6-166)$ & $18.0(2.8-126)$ \\
\hline \multicolumn{4}{|l|}{ Dosage (Gy) } \\
\hline 20 & $4(2.1)$ & $1(0.9)$ & $3(3.7)$ \\
\hline 22 & $9(4.6)$ & $3(2.7)$ & $6(7.4)$ \\
\hline 23 & $4(2.1)$ & $4(3.5)$ & 0 \\
\hline 24 & 75 (38.7) & $43(38.1)$ & $32(39.5)$ \\
\hline 25 & $1(0.5)$ & 0 & $1(1.2)$ \\
\hline 26 & $92(47.4)$ & $54(47.8)$ & $38(46.9)$ \\
\hline 42 & $1(0.5)$ & $1(0.9)$ & 0 \\
\hline 45 & $8(4.1)$ & $7(6.2)$ & $1(1.2)$ \\
\hline
\end{tabular}

Values are presented as number (\%) unless otherwise indicated.

Table 3. Response evaluation

\begin{tabular}{lc} 
Best response according to RECIST & No. $(\%)(\mathbf{n = 1 6 4 )}$ \\
Complete response & $107(65.2)$ \\
Partial response & $22(13.4)$ \\
Stable disease & $27(16.5)$ \\
Progressive disease & $9(5.5)$ \\
Overall response rate & $129(78.7)$ \\
Local disease control rate & $156(94.1)$ \\
\hline
\end{tabular}

RECIST, Response Evaluation Criteria in Solid Tumors as best-overall response.

quent primaries.

The majority of the 194 lesions ( $\mathrm{n}=185,95.4 \%)$ were treated using a single session approach with 20-26 Gy as single dose. More details are shown in Table 2.

Follow-up time was 30.0 months (95\% confidence interval [CI], 19.3 to 40.8). One hundred sixty-four lesions had a tumor evaluation according to RECIST (Table 3). In short, the overall response rate was excellent reaching $78.2 \%$ with only nine lesions (5.5\%) showing progression as best response to treatment. A total of $93(74 \%)$ of tumors recurred with $11(9 \%)$ within the treated area and $84(65 \%)$ outside the treated area.

Median OS was 35.2 months (95\% CI, 22.1 to 48.3) with a 3 - and 5-year survival rate of $47.7 \%$ and $32.5 \%$, respectively. Local median PFS time is 30.7 months (95\% CI, 24.2 to 37.2) and median overall PFS time is 11.9 months $(95 \% \mathrm{CI}, 7.3$ to 16.4).

Survival times (OS and overall PFS) were comparable for $\mathrm{mCRC}$ and non-mCRC patients reaching 32.5 months and 46.2 months in median OS and 9.1 months and 11.9 months for overall PFS (Fig. 1A and B). Size of the treated lesion mattered for OS with a significant shorter median OS for lesions $>5 \mathrm{~cm}$ in diameter (log-rank test $\mathrm{p}=0.032)$ (Fig. $1 \mathrm{C}$ and $\mathrm{D})$. 

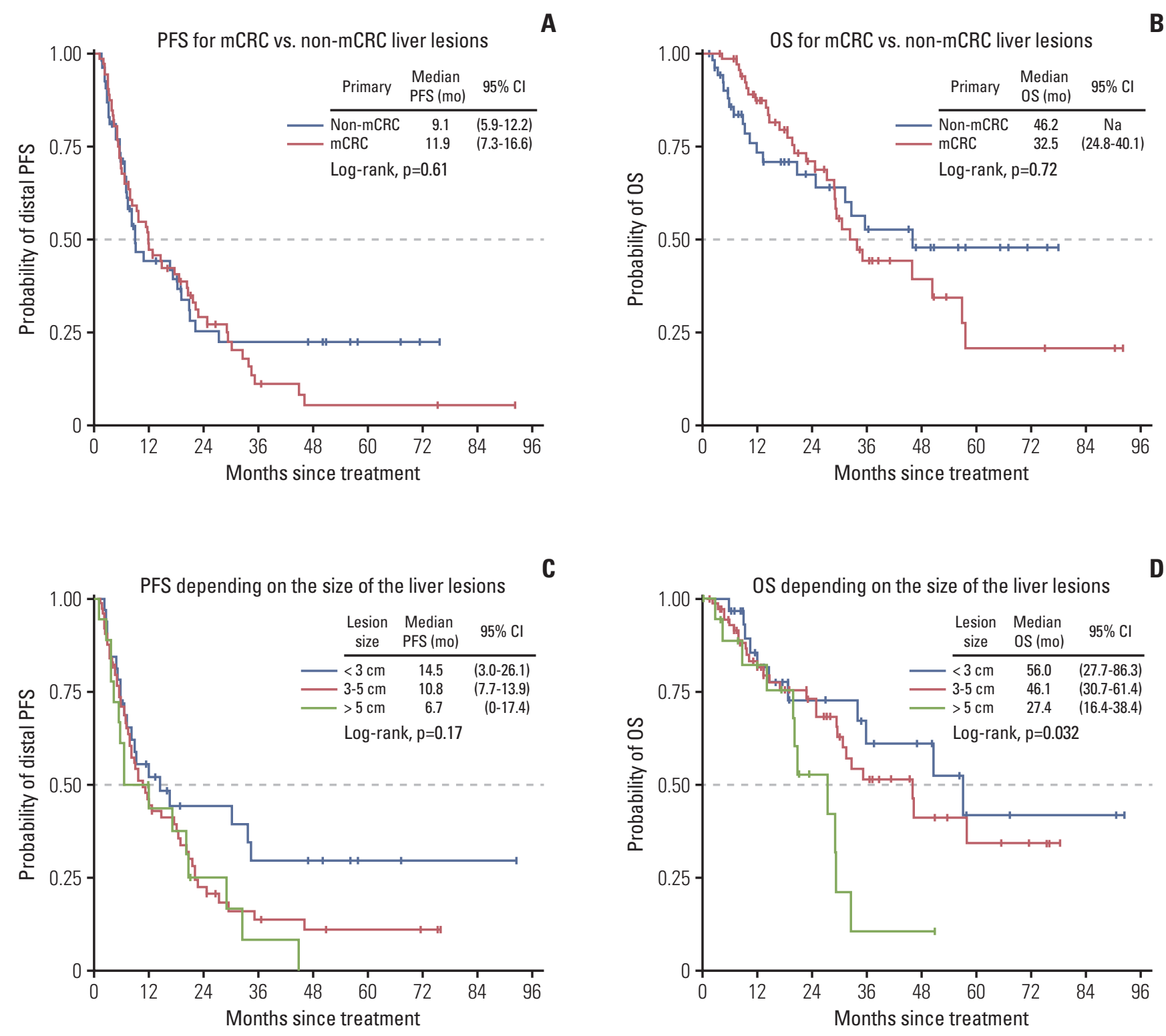

Fig. 1. Survival times according to the primary tumor entity $(A, B)$ and the size of the lesion $(C, D)$. mCRC, colorectal cancer; non-mCRC, lesion not deriving from CRC; OS, overall survival; PFS, progression-free survival; $95 \%$ CI, 95\% confidence interval.

\section{Discussion}

The use of local treatment for liver lesion is a fast developing field. Local modalities, with the exception of surgery, are discussed to be equal-effective in the hand of an experienced therapist. Surgical removal of the tumorous tissue is seen as the gold-standard and probably due to ethical considerations no formal comparison to any local ablative treatment modality has been conducted so far. Local ablative modalities are, when compared to surgery, usually regarded to be less invasive and therefore associated with lower morbidity. The term "toolbox" for the different local treatment modality has been introduced [2] to imply that the possibilities are dependent on the experience and the availability at the respective institution and have comparable outcomes in terms of efficacy.

Radiation therapy has had a limited role in the treatment of liver metastases because of the low tolerance of liver tissue to irradiation [15]. A safe radiation treatment of liver metastases should be possible with a technique that delivers a very 
conformal radiation dose to the tumor and a minimal radiation dose to surrounding critical tissues. This has been achieved with the invention of SBRT in combination with IMRT.

SBRT using the CyberKnife device enables precise delivery of high-dose in a single or a few fractions, with tumor ablation and maximal normal-tissue sparing. Due to the high accuracy using robotic real time tracking techniques even a single session radiation has become possible [12]. Due to the difficulties to calculate the effective dose using the linearquadratic model [16] for radiobiological efficacy doses of 2,000-2,600 cGy were used in single session based on empiric data and a few dose escalation trials [17-20]. Prior phase I/II studies by Herfarth et al. $[17,18]$ investigated single session radiation and have shown higher efficacy with doses from 2,200-2,600 cGy when compared to doses from 1,400-2,200 cGy without a higher incidence of adverse events. The low adverse event rate in our analysis validates this finding. Both, the local control rate of $95.4 \%$ and the overall response rate of $78.2 \%$ proof the concept of ablative single session radiation as a high efficacious low morbidity method to treat liver lesion in curative intent.

The OS in the current study is with a median of 35 months and a 5 -year survival rate of $32.5 \%$ within the findings for surgical resection which is reported to be between $28 \%$ and $58 \%$ for CRLM $[3,21,22]$. A single institution analysis showed a 5-year survival rate of 38\%-39\% for previously untreated CRLM translating into a median OS of 47-48 months [23,24]. The longer OS in the previously untreated group possibly reflects the shorter time between diagnosis and surgery in this favorable cohort of patients when compared to our study of multi-modal pretreated patients. The association of the interval of diagnosis and surgery of CRLM and OS has also been demonstrated in a lager retrospective analysis of more than 600 patients [25]. In the current analysis, heavily pretreated patient underwent SBRT which most likely represents a negative selection with regard to OS. A prospective randomized trial of SBRT vs surgery for patients with limited liver metastases would be highly desirable.

The overall recurrence rate of $74 \%$ is within the previously published data for surgery [24] and demonstrates the curative effect achieved by single-session SBRT using the
CyberKnife system. As it is the case for surgically removed metastases, most of the recurrence $(88 \%, 82 / 93)$ happened outside the treated volume [24]. PFS and OS are therefore dependent for the most on recurrent disease outside the treated region. Notably, one fourth of the treated patients did not show recurrent disease at any site, proving the potential curative value of SBRT.

Similar to thermal ablative techniques [26,27], which may be the biggest competitor to SBRT as a local treatment option; OS after CyberKnife treatment is dependent on the size of the treated lesions. The lower OS rate of patients with lesions $>5 \mathrm{~cm}$ in diameter suggests less efficacy of CyberKnife in larger lesion when treated with single session SBRT. For RFA, the maximal diameter of the treated lesion should not exceed $3 \mathrm{~cm}$ in diameter. Therefore, there may be an advantage for SBRT in lesion of $3-5 \mathrm{~cm}$ in diameter. This is also reflected by the finding that most $(71.1 \%)$ of the treated lesion in our cohort were larger than $3 \mathrm{~cm}$ in diameter. The maximum diameter of liver lesions to be treated by SBRT seems to be $5 \mathrm{~cm}$ as Goodman et al. [20] have also reported the feasibility of single fraction SBRT for lesions up to $5 \mathrm{~cm}$. Furthermore, tissue cooling by vessel flow has been described to reduce the extent of thermally (RFA and MW) induced coagulation [28] and again would favor SBRT over thermal ablative techniques.

This is the largest cohort of SBRT treated liver lesions using the CyberKnife device with a long-term follow-up. The current analysis validates the previously published data of an excellent local control reached by this technique with only minor side effects. OS and PFS are for the most associated with distant recurrence of the underlying disease. This is in line with surgery or other local treatment modalities emphasizing the importance of systemic treatment in addition to local ablative modalities for the majority of the patients. Robotic radiosurgery using a single session approach has a high efficacy to control the radiated lesion with the potential to cure patients.

\section{Conflicts of Interest}

Conflict of interest relevant to this article was not reported.

\section{References}

1. Kopetz S, Chang GJ, Overman MJ, Eng C, Sargent DJ, Larson $\mathrm{DW}$, et al. Improved survival in metastatic colorectal cancer is associated with adoption of hepatic resection and improved chemotherapy. J Clin Oncol. 2009;27:3677-83.
2. Van Cutsem E, Cervantes A, Adam R, Sobrero A, Van Krieken $\mathrm{JH}$, Aderka D, et al. ESMO consensus guidelines for the management of patients with metastatic colorectal cancer. Ann Oncol. 2016;27:1386-422. 
3. Abdalla EK, Vauthey JN, Ellis LM, Ellis V, Pollock R, Broglio $\mathrm{KR}$, et al. Recurrence and outcomes following hepatic resection, radiofrequency ablation, and combined resection/ablation for colorectal liver metastases. Ann Surg. 2004;239:818-25.

4. Chan KM, Wu TH, Cheng CH, Lee WC, Chiang JM, Chen JS, et al. Prognostic significance of the number of tumors and aggressive surgical approach in colorectal cancer hepatic metastasis. World J Surg Oncol. 2014;12:155.

5. Park MS, Yi NJ, Son SY, You T, Suh SW, Choi YR, et al. Histopathologic factors affecting tumor recurrence after hepatic resection in colorectal liver metastases. Ann Surg Treat Res. 2014;87:14-21.

6. van Amerongen MJ, Jenniskens SF, van den Boezem PB, Futterer JJ, de Wilt JH. Radiofrequency ablation compared to surgical resection for curative treatment of patients with colorectal liver metastases: a meta-analysis. HPB (Oxford). 2017;19: 749-56.

7. Park EK, Kim HJ, Kim CY, Hur YH, Koh YS, Kim JC, et al. A comparison between surgical resection and radiofrequency ablation in the treatment of hepatocellular carcinoma. Ann Surg Treat Res. 2014;87:72-80.

8. Santambrogio R, Chiang J, Barabino M, Meloni FM, Bertolini E, Melchiorre F, et al. Comparison of laparoscopic microwave to radiofrequency ablation of small hepatocellular carcinoma $(\leq 3 \mathrm{~cm})$. Ann Surg Oncol. 2017;24:257-63.

9. Rusthoven KE, Kavanagh BD, Cardenes H, Stieber VW, Burri SH, Feigenberg SJ, et al. Multi-institutional phase I/II trial of stereotactic body radiation therapy for liver metastases. J Clin Oncol. 2009;27:1572-8.

10. Dawood O, Mahadevan A, Goodman KA. Stereotactic body radiation therapy for liver metastases. Eur J Cancer. 2009;45: 2947-59.

11. Pech M, Mohnike K, Wieners G, Bialek E, Dudeck O, Seidensticker $\mathrm{M}$, et al. Radiotherapy of liver metastases: comparison of target volumes and dose-volume histograms employing CT- or MRI-based treatment planning. Strahlenther Onkol. 2008;184:256-61.

12. Stintzing S, Hoffmann RT, Heinemann V, Kufeld M, Rentsch M, Muacevic A. Radiosurgery of liver tumors: value of robotic radiosurgical device to treat liver tumors. Ann Surg Oncol. 2010;17:2877-83.

13. Benedict SH, Yenice KM, Followill D, Galvin JM, Hinson W, Kavanagh B, et al. Stereotactic body radiation therapy: the report of AAPM Task Group 101. Med Phys. 2010;37:4078-101.

14. Schweikard A, Shiomi H, Adler J. Respiration tracking in radiosurgery. Med Phys. 2004;31:2738-41.

15. Dawson LA, Normolle D, Balter JM, McGinn CJ, Lawrence TS, Ten Haken RK. Analysis of radiation-induced liver disease using the Lyman NTCP model. Int J Radiat Oncol Biol Phys. 2002;53:810-21.

16. Kirkpatrick JP, Brenner DJ, Orton CG. Point/Counterpoint.
The linear-quadratic model is inappropriate to model high dose per fraction effects in radiosurgery. Med Phys. 2009;36: 3381-4.

17. Herfarth KK, Debus J, Lohr F, Bahner ML, Rhein B, Fritz P, et al. Stereotactic single-dose radiation therapy of liver tumors: results of a phase I/ II trial. J Clin Oncol. 2001;19:164-70.

18. Herfarth KK, Hof H, Bahner ML, Lohr F, Hoss A, van Kaick $\mathrm{G}$, et al. Assessment of focal liver reaction by multiphasic CT after stereotactic single-dose radiotherapy of liver tumors. Int J Radiat Oncol Biol Phys. 2003;57:444-51.

19. Wagman R, Yorke E, Ford E, Giraud P, Mageras G, Minsky B, et al. Respiratory gating for liver tumors: use in dose escalation. Int J Radiat Oncol Biol Phys. 2003;55:659-68.

20. Goodman KA, Wiegner EA, Maturen KE, Zhang Z, Mo Q, Yang G, et al. Dose-escalation study of single-fraction stereotactic body radiotherapy for liver malignancies. Int J Radiat Oncol Biol Phys. 2010;78:486-93.

21. Nordlinger B, Guiguet M, Vaillant JC, Balladur P, Boudjema $\mathrm{K}$, Bachellier $\mathrm{P}$, et al. Surgical resection of colorectal carcinoma metastases to the liver: a prognostic scoring system to improve case selection, based on 1568 patients. Association Francaise de Chirurgie. Cancer. 1996;77:1254-62.

22. Fong Y, Fortner J, Sun RL, Brennan MF, Blumgart LH. Clinical score for predicting recurrence after hepatic resection for metastatic colorectal cancer: analysis of 1001 consecutive cases. Ann Surg. 1999;230:309-18.

23. Silberhumer GR, Paty PB, Denton B, Guillem J, Gonen M, Araujo RL, et al. Long-term oncologic outcomes for simultaneous resection of synchronous metastatic liver and primary colorectal cancer. Surgery. 2016;160:67-73.

24. Homayounfar K, Bleckmann A, Conradi LC, Sprenger T, Lorf $\mathrm{T}$, Niessner $\mathrm{M}$, et al. Metastatic recurrence after complete resection of colorectal liver metastases: impact of surgery and chemotherapy on survival. Int J Colorectal Dis. 2013;28:100917.

25. Leal JN, Bressan AK, Vachharajani N, Gonen M, Kingham TP, D'Angelica MI, et al. Time-to-surgery and survival outcomes in resectable colorectal liver metastases: a multi-institutional evaluation. J Am Coll Surg. 2016;222:766-79.

26. Veltri A, Sacchetto P, Tosetti I, Pagano E, Fava C, Gandini G. Radiofrequency ablation of colorectal liver metastases: small size favorably predicts technique effectiveness and survival. Cardiovasc Intervent Radiol. 2008;31:948-56.

27. Berber E, Siperstein A. Local recurrence after laparoscopic radiofrequency ablation of liver tumors: an analysis of 1032 tumors. Ann Surg Oncol. 2008;15:2757-64.

28. Lencioni R, de Baere T, Martin RC, Nutting CW, Narayanan G. Image-guided ablation of malignant liver tumors: recommendations for clinical validation of novel thermal and nonthermal technologie. A Western Perspective. Liver Cancer. 2015;4:208-14. 